\title{
Clinical Commentary: Rehabilitation Using Acute Dry Needling for Injured Athletes Returning to Sport and Improving Performance
}

\author{
Tyler J. Gregory, P.T., D.P.T., Samuel A. Rauchwarter, P.T., D.P.T., S.C.S., and \\ Michael D. Feldman, M.D.
}

\begin{abstract}
Dry needling is most commonly used in the subacute and chronic phases of an injury; therefore, it is imperative to understand the use of dry needling in the acute phases of an injury. There are four main reasons to use dry needling during the acute phase of injury: to relieve pain, decrease edema, increase range of motion and flexibility, and increase strength and power. Dry needling can be used pregame, postgame, during half time or an intermission, or following an injury. Although further research is needed, acute dry needling can be used to bolster athletes' health and possibly improve sports performance.
\end{abstract}

\section{Introduction}

D ry needling is defined as the insertion of solid filiform needles into the body based on an understanding of anatomy and physiology. ${ }^{1}$ Dry needling is focused on targeting muscle, neural, and connective tissues. ${ }^{1}$ Dry needling performed by a physical therapist and other health care professionals has become common in the United States over the last couple of decades. $^{2}$

\section{History}

The technique for dry needling has evolved over the last 70 years. $^{1}$ In the early literature regarding dry needling, it was not specified which type of needle was used. This has led to ongoing debate about the origins of

From the Sports Resident Physical Therapist, BodyCentral Physical Therapy, Tucson, Arizona (T.J.G.); BodyCentral Physical Therapy, Tucson, Arizona (S.A.R.); and Department of Sports Medicine, Banner-University Medical Center and Department of Orthopedic Surgery, University of Arizona School of Medicine, Tucson, Arizona (M.D.F.)

The authors report no conflicts of interest in the authorship and publication of this article. Full ICMJE author disclosure forms are available for this article online, as supplementary material.

Received August 2, 2021; accepted September 15, 2021.

Address correspondence to Tyler J. Gregory, P.T., D.P.T., Sports Resident Physical Therapist, BodyCentral Physical Therapy, 3501 E Speedway Blvd \#145, Tucson, AZ 85716,U.S.A. E-mail: tylergregory@bodycentralpt.net

(C) 2021 THE AUTHORS. Published by Elsevier Inc. on behalf of the Arthroscopy Association of North America. This is an open access article under the CC BY-NC-ND license (http://creativecommons.org/licenses/by-nc-nd/4.0/).

2666-061X/211105

https://doi.org/10.1016/j.asmr.2021.09.035 dry needling. ${ }^{2}$ Many individuals consider Janet Travell, the former personal physician to presidents John F. Kennedy and Lydon B Johnson, to be the founder of dry needling. ${ }^{2}$ This is debated heavily mostly because of a lack of specificity in the type of needle used in the technique. ${ }^{2}$ Later detailed in a 1983 book, Myofascial Pain and Dysfunction: The Trigger Point Manual, the concept of injections is discussed, allowing one to surmise that hypodermic needles are being discussed. ${ }^{2}$ This is seen in some other articles published up until about 1980s. ${ }^{2}$ The use of hypodermic needles for dry needling is not considered dry needling at this current time.

Currently, injection-based therapies using hollow needles are termed wet needling. ${ }^{1}$ Wet needling is used to inject a variety of substances ranging from corticosteroids to botulinum toxins. ${ }^{1}$ Physical therapists, aside from some in the military, do not perform injections. One of the earliest known articles describing dry needling using a solid filiform needle was published in 1979. ${ }^{2}$ Dry needling started receiving widespread popularity in the early 21 st century. ${ }^{2}$

\section{Legal Regulations}

Currently dry needling is practiced in Europe, Australia, and in some states in the United States of America. ${ }^{2}$ In the United States, the ability of physical therapists to implement dry needling is based on state practice acts, which varies from state to state. ${ }^{1}$ This means in some states physical therapist are unable to practice dry needling, while in other states they are. Physical therapists are able to engage in dry needling after having taken a 
comprehensive continuing education course. ${ }^{2}$ The ability to perform dry needling, the structures in which the needle is being inserted, and the specific techniques used are limited by state practice acts. ${ }^{1}$ To summarize, physical therapists ability to dry needle is heavily dependent on the state in which they practice.

\section{Dry Needling Versus Acupuncture}

Often debated is whether acupuncture and dry needling are different and what are those differences. These waters are muddied further by significant scholarly literature using the terms dry needling and acupuncture interchangeably. ${ }^{1}$ Complicating the debate further is that there are multiple forms of acupuncture. The two main groups of acupuncture are traditional acupuncture and Western medical acupuncture.

Traditional acupuncture originated in China about 2,000 years ago. ${ }^{2}$ In traditional acupuncture, the goal is to move qi along meridians and to balance yin and yang. ${ }^{1,2}$ Western medical acupuncture is very similar to dry needling with minor differences in theory. Both dry needling and Western medical acupuncture are rooted in contemporary knowledge of anatomy and physiology and are both done by health care professionals. ${ }^{2}$ One area of theoretical difference is that Western medical acupuncture will target ah shi points, while dry needling targets trigger points. ${ }^{2}$ Ah shi points are acupuncture points that are painful with palpation. ${ }^{2}$ Both of these targets have similar definitions and distributions. $^{1,2}$ It can be argued that in implementation, these different treatment modalities have more in common than not, but the major difference is the school of thinking and reasoning.

\section{When, Why, and How Dry Needling Is Used}

Dry needling is used to treat a variety of anatomic structures and for a multitude of reasons. Dry needling is used to stimulate neural, muscular, and connective tissues. ${ }^{1}$ This includes scar tissue, tendons, and muscles (Fig 1). Some pathologies that have shown benefit from receiving dry needling include knee osteoarthritis, piriformis syndrome, and plantar fasciitis. ${ }^{1}$ It is most common to see subacute and chronic conditions receive dry needling by a physical therapist. This may be due to the delay in time it takes a patient to see a physical therapist. Another possible explanation is that many physical therapists try to use other minimally invasive techniques before progressing along the continuum. This highlights the need to further investigate and discuss the possibility of dry needling in acute conditions and the athletes who hope to receive its shortterm healing benefits.

\section{Effects of Dry Needling}

Dry needling can have biomechanical, endocrinological, and vascular effects. The literature states that microcirculation, short-term pain, nerve conduction velocity, sensory nerve conduction velocity, collagen proliferation, local oxygen, range of motion, and muscular strength saturation all improve with dry needling. ${ }^{1,2}$ When electrical stimulation is used, evidence shows an increase in $\beta$-endorphins and a decrease cortisol. ${ }^{1}$ The main mechanisms for the effects noted previously are attributed to manipulation of gate control theory, endogenous opioid system, and purinergic signaling system. ${ }^{2}$

\section{Physical Therapists in Sport}

Sports physical therapists have a role to play in the management of athletes and athletic related injuries. ${ }^{3}$ Sports certified specialist (SCS) is a credential earned through time spent rehabilitating athletes, learning about acute management, and performing sideline coverage among other requirements. At a minimum, the additional training required should comprise an emergency responder certification. ${ }^{3}$ Physical therapists without additional training are not qualified to provide sideline coverage. ${ }^{3}$ Physical therapists who have obtained their SCS certification are able to perform not only rehabilitation of athletes, but also manage athletes from the moment of injury with sideline coverage and prehospital care as needed. ${ }^{3}$ Sports physical therapy residency programs educate the resident on how to provide care for athletes with the goal of having these residents receive their SCS certification. There is a growing use of dry needling in sport medicine. ${ }^{2}$ Sports physical therapists are in a privileged position to bring dry needling to athletes anywhere in their healing continuum.

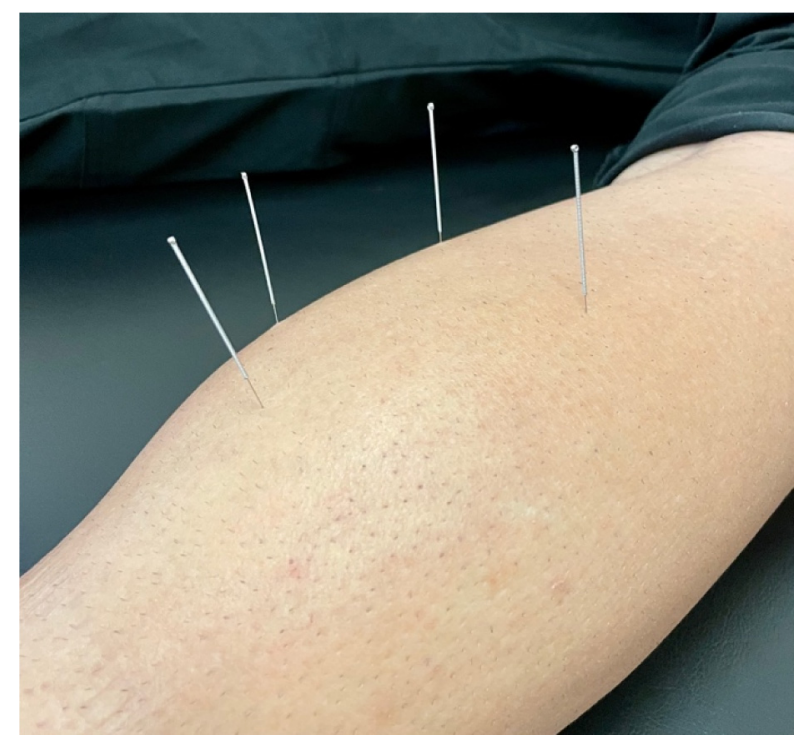

Fig 1. Example of dry needle application to the right calf musculature. Photo taken by Samuel Rauchwarter, P.T., D.P.T., S.C.S. 


\section{Main Reasons to Implement Dry Needling}

In our opinion, there are four main reasons to use dry needling during the acute phase: to relieve pain, decrease edema, increase range of motion and flexibility, and increase strength and power. Focusing specifically on immediate, short term, and acute effects of dry needling in scholarly literature will allow us to further extrapolate how these effects can be used in practice of a sports physical therapist to benefit an athlete under their care.

\section{Pain}

Improvements in pain are one of the most often reported findings following dry needling treatment, although the degree of lasting effect varies. In an article by Mejuto-Vasquez et al., subjects with acute neck pain were treated with trigger point dry needling. ${ }^{4}$ At 10 minutes and 1 week after dry needling, the participants had decreased pain intensity and an increased pressure pain threshold when compared to the control group. ${ }^{4}$ Similarly, a case report by Pakovich ${ }^{5}$ looked at treating acute neck pain in an active 64-year-old female with dry needling and found significant improvements in pain and disability following intervention. ${ }^{5}$ The subject's pain and functional improvements were seen after the initial treatment, which lasted until the next treatment session, one month later. ${ }^{5}$ If lasting effects from one session of dry needling are seen for up to a month, it may be worth suggesting the use of dry needling prior to a game or even in the subacute phase of an injury in the week leading up to a game.

In a Cochrane Review by Furlan et al., ${ }^{6}$ subjects with chronic low back pain had relief and improved function in the short-term. ${ }^{6}$ Many athletes suffer from chronic low back pain, and we can infer that this might negatively impact their performance. In another study by Kamali et al., ${ }^{7} 40$ overhead athletes with unilateral shoulder impingement syndrome received dry needling to trigger points in the upper trapezius and infraspinatus muscles. Dry needling of trigger points in the upper trapezius and infraspinatus both resulted in statistically significant decreases in pain intensity and disability. ${ }^{7}$ For example, these results may translate to clinical practice when using dry needling on a baseball pitcher with shoulder pain post-game. In a recent study, a group of subjects who had an acute ankle sprain that resulted in chronic instability saw decreased pain and improved function following a single dry needling session. ${ }^{8}$

\section{Range of Motion}

Mejuto-Vasquez et al. ${ }^{4}$ found significant improvements in cervical range of motion at 10 minutes and 1 week postintervention. ${ }^{4}$ In a systematic review, Bynum et al. ${ }^{9}$ found strong evidence that dry needling improved cervical range of motion both actively and passively. ${ }^{9}$ Dry needling has been shown to increase athletes range of motion. In a 2013 article by Dembowski et al., dry needling was used in conjunction with eccentric exercises to return a collegiate pole vaulter with a hamstring strain to sport. The college pole vaulter was seen 3 times a week for 3 weeks with dry needling once a week. ${ }^{10}$ By day 12 , the subject has symmetrical active straight leg raise and no longer had a deficit in active range of motion. ${ }^{10}$ Returning an athlete to full range of motion may aid in their ability to progress through rehabilitation and eventually return to sport. This article demonstrates a more well-rounded treatment approach, including using dry needling as an additive to a comprehensive rehabilitation plan.

A recent study by Alaei et al. found that dry needling improves active knee extension as much as static stretching. ${ }^{11}$ Additionally, passive movement improved greater with dry needling than with static stretching in individuals with hamstring tightness. ${ }^{11}$ In a 2016 case study by Passigli et al., a male dance instructor with subacromial impingement on his dominant side received dry needling to target myofascial trigger points in his posterior shoulder. ${ }^{12}$ Reductions in pain and improvements in range of motion were found following each treatment. ${ }^{12}$ The changes to pain intensity and glenohumeral internal rotation and horizontal adduction were found to be clinically significant. ${ }^{12}$

\section{Strength and Power}

Dry needling has also been shown to increase strength and power in athletes. In a case report by Luna, ${ }^{13}$ traditional acupuncture and Chinese medicine techniques were used to increase range of motion, decrease pain, and increase grip strength in a Brazilian Jiu Jitsu athlete with proximal interphalangeal inflammation. ${ }^{13}$ In healthy college students dry needling improved vertical jump height immediately following intervention. ${ }^{14}$ This brings into question the idea of using dry needling to improve athletic performance. Jankowski et al. investigated the acute effects of dry needling on myofascial trigger points in ballet dancers. They found that left plantar flexion torque increased, but range of motion and pain were unaffected. ${ }^{15}$ Jankowski surmised that further research is needed prior to implementing dry needling prior to athletic activity. ${ }^{15}$ This is just a small sampling of the uses of dry needling with athletes and in the acute setting.

\section{Edema}

The use of dry needling to control edema following an acute injury is scarce in the literature. Acupuncture has been shown to decrease swelling following a total knee arthroscopy in the postacute phase ${ }^{16}$ In a 2012 article, Mikashima et al. found that the group receiving acupuncture treatment saw a reduction in pain and returned to preoperative range of motion earlier than a 
control group. ${ }^{17}$ In a study by Cassileth et al., they looked at acupuncture in the treatment of breast cancer-related lymphedema. They found that acupuncture may reduce arm circumference. Eleven of 33 patients saw greater than $33 \%$ limb reduction with the mean circumference reduction being $.90 \mathrm{~cm}^{.17}$ Increasingly, research on dry needling with athletes is appearing in the academic literature, but not enough at this time to draw conclusive findings. ${ }^{18}$

\section{Pregame}

Dry needling can be used in a variety of scenarios in the acute phase of an injury. These scenarios include pregame and in-game, postgame, and in the rehab setting. Dry needling can be used in multiple ways for the pregame and in-game scenarios. One way to treat with dry needling pregame is for muscle activation. This can be used as a passive warm-up with an in-and-out technique. This technique can be described as inserting the needle into the targeted muscle to elicit a response and taking the needling out right away. This can be considered a passive warm up to athletic competition, but it should be used in conjunction with a dynamic on-field warm-up to ensure the athlete is ready to perform. In-and-out technique can also be used in a cramping or spasming muscle during the game. The current belief is that a needle will reset the muscle and allow the muscle to relax.

\section{Post-Game}

Dry needling can also be used after a game for a multitude of effects in an acute injury setting. The main reason to use dry needling after a competition would be for pain modulation. One way to use dry needles for pain modulation is to use them on trigger points. This can be done with the in-and-out technique or with leaving the needle in for a set period of time. Leaving the needle in for a set period of time can help to downregulate the nervous system after competition. Depending on what you are trying to treat, the needle can be left in for various amounts of time. Treating a certain joint or segment of the body may call for the needle to be left in for 10 minutes. ${ }^{19,20}$ When treating systemically, leaving the needles in for 20-30 minutes is optimal. ${ }^{19,20}$ The needles can be left in with or without electronic stimulation (e-stim).

Stimulation should start with the parameters of $2 \mathrm{~Hz}$ to release enkephalins, $\beta$-endorphins and endomorphins. ${ }^{21-24}$ This will provide longer-lasting pain relief but with a slower onset of pain modulation. If the athlete is experiencing stronger pain signals, a setting of $100 \mathrm{~Hz}$ can be used for short-lived pain relief with a more immediate effect. ${ }^{19,20}$

Another way to downregulate the nervous system is to use primary passive trigger points (PPTP), which is based off the work of Houchi Dung. ${ }^{25}$ Dung describes these trigger points as areas of increased sensitivity in certain times of our lives. He has been able to identify 6 points in the upper extremity, 9 points in the lower extremity, 7 points in the spine, and 3 points in the face that can provide athletes with a downregulation of the nervous system to allow for better recovery. For the purposes of this article, we will not go into depth about Dr. Dung's work beyond what has been noted above.

\section{Acute Management of Injury}

Dry needling can also be used for the acute management of injury in the rehabilitation setting. It can be used for pain modulation as noted above but also be used for muscle activation, edema control, and trigger point release. For muscle activation, one may use the in-and-out technique, but what is more common is leaving the needles in and using electronic stimulation (e-stim) for muscle activation. The e-stim maybe be set at $2 \mathrm{~Hz}$ or $100 \mathrm{~Hz}$ setting for no more than $5 \mathrm{mi}-$ nutes. ${ }^{19,20}$ The idea is to excite the muscle fibers and branches of the motor nerves to create a contraction. This is often used in patient cases involving nerve damage or 6 or more weeks out after an operation when the patient is having trouble getting a strong muscular contraction.

\section{Edema Control}

Dry needling may also be used for edema control. The provider will place a multitude of needles around the injured site. These needles are often shorter 13-mm needles, and they are left in for up to 30 minutes. ${ }^{19,20}$ This can be used for both acute and chronic swelling. This technique has not been well researched at this time.

\section{Trigger Point Release}

Trigger point release can be done effectively with dry needles. This technique involves the provider placing a needle directly into a trigger point to help release that trigger point. This is normally done by leaving the needle in the trigger point for 10 or more minutes. ${ }^{19,20}$ The provider may also use a piston maneuver to help release the trigger point. A piston maneuver is a form of needle manipulation where the needle is placed into the skin and is moved in an up and down fashion while keeping the needle in the skin. This can be more painful for the athlete but can also be more effective in making multiple lesions and releasing the trigger point.

\section{Conclusion}

In conclusion, dry needling can be used to help treat edema, to improve range of motion, to improve neuromuscular firing of a muscle, and to relieve pain. Dry needling has been used commonly in the subacute and chronic phases of an injury but has not been researched in the acute phase. On the basis of clinical 
experience and current research using dry needling for acute injury, it has been helpful for athletes. More research is needed to confirm the effectiveness. Although there is little research that shows the effects of acute dry needling, medical providers should still consider this modality to help treat their patients in the acute phase of an injury.

\section{References}

1. Dunning J, Butts R, Mourad F, Young I, Flannagan S, Perreault T. Dry needling: A literature review with implications for clinical practice guidelines. Phys Ther Rev 2014; 19:252-265.

2. Zhou K, Ma Y, Brogan MS. Dry needling versus acupuncture: The ongoing debate. Acupunct Med 2015;33:485-490.

3. Smith D. Are all physical therapists qualified to provide sideline coverage of athletic events? Int J Sports Phys Ther 2012;7:120-123.

4. Mejuto-Vázquez MJ, Salom-Moreno J, OrtegaSantiago R, Truyols-Domínguez S, Fernández-de-LasPeñas C. Short-term changes in neck pain, widespread pressure pain sensitivity, and cervical range of motion after the application of trigger point dry needling in patients with acute mechanical neck pain: a randomized clinical trial. J Orthop Sports Phys Ther 2014;44:252-260.

5. Pavkovich R. The use of dry needling for a subject with acute onset of neck pain: A case report. Int J Sports Phys Ther 2015;10:104-113.

6. Furlan AD, van Tulder MW, Cherkin D, et al. Acupuncture and dry needling for low back pain. Cochrane Database Syst Rev 2005; 1:CD001351.

7. Kamali F, Sinaei E, Morovati M. Comparison of upper trapezius and infraspinatus myofascial trigger point therapy by dry needling in overhead athletes with unilateral shoulder impingement syndrome. J Sport Rehabil 2019;28: 243-249.

8. Salom-Moreno J, Ayuso-Casado B, Tamaral-Costa B, Sánchez-Milá Z, Fernández-de-las-Peñas C, Alburquerque-Sendín F. Trigger point dry needling and proprioceptive exercises for the management of chronic ankle instability: A randomized clinical trial. Evid Based Complement Alternat Med 2015;2015:e790209.

9. Bynum R, Garcia O, Herbst E, et al. Effects of dry needling on spasticity and range of motion: A systematic review. Am J Occup Ther 2021;75:1-13.

10. Dembowski SC, Westrick RB, Zylstra E, Johnson MR. Treatment of hamstring strain in a collegiate pole-vaulter integrating dry needling with an eccentric training program: A resident's case report. Int J Sports Phys Ther 2013;8:328-340.

11. Alaei P, Ansari NN, Naghdi S, Fakhari Z, Komesh S, Dommerholt J. Dry needling for hamstring flexibility: A single-blind randomized controlled trial. J Sport Rehabil 2020;30:452-457.

12. Passigli S, Plebani G, Poser A. Acute effects of dry needling on posterior shoulder tightness. A case report. Int J Sports Phys Ther 2016;11:254-263.

13. Luna R. Treatment of joint injuries and inflammation in a professional Brazilian jiu-jitsu athlete. J Am Soc Acupuncturists 2019;6:19-21.

14. Bandy WD, Nelson R, Beamer L. Comparison of dry needling vs. sham on the performance of vertical jump. Int J Sports Phys Ther 2017;12:747-751.

15. Janowski JA, Phelan-Smith DML, Brady MNK, et al. Acute effects of dry needling on myofascial trigger points in the triceps surae of ballet dancers: A pilot randomized controlled trial. Int J Sports Phys Ther 2021;16:418-430.

16. Mikashima Y, Takagi T, Tomatsu T, Horikoshi M, Ikari K, Momohara S. Efficacy of acupuncture during post-acute phase of rehabilitation after total knee arthroplasty. J Tradit Chin Med 2012;32:545-548.

17. Cassileth BR, Van Zee KJ, Yeung KS, et al. Acupuncture in the treatment of upper-limb lymphedema. Cancer 2013;119:2455-2461.

18. Gentil LB. Treatment of sport injuries with acupuncture: A literature review. Rev Bras Med Esporte 2018;24:316-321.

19. Perreault T, Flannagan SO, Grubb MT, Grubb R. Mechanisms and dose parameters of manual needle stimulation: Clinical considerations - Part 2. Acupuncture Physiother, 2019. Autumn:15.

20. Srbely JZ, Dickey JP, Lee D, Lowerison M. Dry needle stimulation of myofascial trigger points evokes segmental anti-nociceptive effects. J Rehab Med 2010;42:463-468.

21. Basbaum AL, Bautista DM, Scherrer G, Julius D. Cellular and molecular mechanism of pain. Cell 2009;139:267-284.

22. Cagnie B, Dewitte V, Barbe T, Timmermans F, Delrue N, Meeus M. Physiologic effects of dry needling Curr Pain Headache Rep 2013;17:348.

23. Gyer G, Michael J, Tolson B. Dry needling for manual therapists. Philadelphia: Singapore Dragon, 2016.

24. Sobrian SK, Walters E. Enhanced satellite cell activity in aging skeletal muscle after manual acupuncture-induced injury." Chinese Med 2014;5:22-33.

25. Dung H. Acupuncture an anatomical approach. Second edition. Boca Raton, FL: CRC Press, 2014. 